\title{
Hard X-ray microtomography of Zebrafish larvae
}

Melissa Osterwalder, Jan Stephan Bolten, Griffin Rodgers, Mattia Humbel, Georg Schulz, et al.

Melissa Osterwalder, Jan Stephan Bolten, Griffin Rodgers, Mattia Humbel, Georg Schulz, Christine Tanner, Jörg Huwyler, Bert Müller, "Hard X-ray microtomography of Zebrafish larvae," Proc. SPIE 11886, International Conference on X-Ray Lasers 2020, 1188614 (8 July 2021); doi: 10.1117/12.2593119

SPIE. Event: XVII International Conference on X-Ray Lasers, 2020, Online Only 


\title{
Hard X-ray Microtomography of Zebrafish Larvae
}

\author{
Melissa Osterwalder*a,b, Jan Stephan Bolten ${ }^{\mathrm{c}}$, Griffin Rodgers ${ }^{\mathrm{a}, \mathrm{b}}$, Mattia Humbel ${ }^{\mathrm{a}}$, \\ Georg Schulz ${ }^{\mathrm{a}, \mathrm{b}, \mathrm{d}}$, Christine Tanner ${ }^{\mathrm{a}, \mathrm{b}}$, Jörg Huwyler ${ }^{\mathrm{c}}$, and Bert Müller ${ }^{\mathrm{a}, \mathrm{b}}$ \\ ${ }^{a}$ Biomaterials Science Center, Department of Biomedical Engineering, University of Basel, \\ Gewerbestrasse 14, 4123 Allschwil, Switzerland \\ ${ }^{b}$ Biomaterials Science Center, Department Clinical Research, c/o University Hospital Basel, \\ Schanzenstrasse 55, 4031 Basel, Switzerland \\ ${ }^{\mathrm{c}}$ Division of Pharmaceutical Technology, Department of Pharmaceutical Sciences, University of \\ Basel, Klingelbergstrasse 50, 4056 Basel, Switzerland \\ ${ }^{\mathrm{d} C o r e ~ F a c i l i t y ~ M i c r o-~ a n d ~ N a n o t o m o g r a p h y, ~ D e p a r t m e n t ~ o f ~ B i o m e d i c a l ~ E n g i n e e r i n g, ~}$ \\ University of Basel, Gewerbestrasse 14, 4123 Allschwil, Switzerland
}

\begin{abstract}
Hard X-ray micro computed tomography can be used for three-dimensional histological phenotyping of zebrafish embryos down to $1 \mu \mathrm{m}$ or below without the need for staining or physical slicing. Current advances in zebrafish embryo imaging, however, mostly rely on synchrotron radiation sources or highly advanced laboratory sources, which despite their evident strengths with regard to their beam properties and the implementation of phase contrast imaging techniques, lack accessibility. Therefore, we evaluated the performance of a conventional SkyScan 1275 laboratory $\mu \mathrm{CT}$ scanner in absorption contrast mode for the visualization of anatomical features in ethanol- and paraffin-embedded zebrafish embryos. We compare our results to readily available synchrotron data where 35 anatomical structures were identified. Despite having a more than ten times larger voxel length, approximately two thirds of the features could also be determined with laboratory microtomography. This could allow to monitor morphological changes during development or disease progression on large sample numbers, enabling the performance of preclinical studies in a local laboratory.
\end{abstract}

Keywords: Zebrafish embryo, hard X-ray computed tomography, microCT, conventional X-ray source, histological phenotyping, ethanol and paraffin embedding

\section{INTRODUCTION}

The zebrafish embryo is a well suited vertebrate model for various biomedical disciplines, with its starting point before the $21^{\text {st }}$ century in the fields of transgenic research, early toxicity and drug discovery screenings. ${ }^{1,2}$ Since then, zebrafish experiments have evolved and found their application even in nanomedicine research, i.e. to analyze the pharmacokinetics, biodistribution and targeting of nanocarriers ${ }^{3-5}$ as well as the developmental toxicity of drugs. ${ }^{6}$ Zebrafish are a reliable in vivo model, since their genetic profile shares $70 \%$ similarity with humans. ${ }^{7}$ Toxicological readouts such as survival, hatching rate or organ malformations can be assessed in a fast and controlled manner. ${ }^{8}$ Further, zebrafish embryos have many practical advantages as they exhibit a rapid embryogenesis and a fast reproduction capability. They are simple to handle, small in size and by adding bleaching substance (e.g. 1-phenyl-thiourea), ${ }^{9}$ they remain optically transparent during their early developmental stages. ${ }^{10,11}$

Nowadays, the majority of zebrafish embryo research still relies on fluorescence imaging techniques such as confocal laser scanning fluorescence microscopy. However, these techniques depend on fluorophore staining and are limited by the penetration depth of visible light when investigating larvae at later developmental time points. These limitations can be overcome by X-ray microtomography, which due to the high penetration depth of hard $\mathrm{X}$-rays allows for three-dimensional whole animal imaging with spatial resolutions down to $1 \mu \mathrm{m}$ in a labelfree manner and without the need for physical sectioning. While the beam properties of synchrotron radiation

*melissa.osterwalder@unibas.ch, phone: +41 6120754 38, www.bmc.unibas.ch 
sources are desirable, providing highly brilliant radiation with a high flux and brightness and a spatially coherent beam that allows for phase contrast X-ray imaging techniques, the user accessibility is restricted. Therefore, it is eminently desirable to explore the feasibility of X-ray imaging of zebrafish embryos with laboratory sources, which provide accessibility for local users.

Synchrotron radiation-based micro computed tomography ( $\mathrm{SR} \mu \mathrm{CT}$ ) has been used for the three-dimensional histotomography of whole zebrafish embryos and 33 days post fertilization (dpf) juvenile zebrafish, providing three-dimensional renderings as well as organ sectionings and showing that cellular architecture can be extracted throughout the entire organism. ${ }^{12}$ Further, a recent study has demonstrated the performance of SR $\mu \mathrm{CT}$ for the localization of injected superparamagnetic iron oxide nanoparticles in zebrafish embryos. ${ }^{13}$ Thereby, the nanoparticle biodistribution was assessed with isotropic resolution around $2 \mu \mathrm{m}$, providing the full anatomical context. Other recent research efforts have successfully implemented advanced laboratory-based X-ray techniques for zebrafish embryo imaging. The morphology of heavy metal-stained zebrafish embryos has been uncovered with micrometer resolution by laboratory-based X-ray NanoCT ${ }^{14}$ and muscle imaging on unstained 20 dpf zebrafish larvae with spatial resolution of 4 to $6 \mu \mathrm{m}$ has been demonstrated with a liquid-metal-jet microfocus X-ray source by propagation-based X-ray phase contrast tomography. ${ }^{15}$ In addition, functional biomedical imaging has been performed on zebrafish embryos by X-ray fluorescence microtomography with synchrotron radiation, providing a three-dimensional visualization of the elemental densities of trace metals within cells and tissue sections during embryonic development. ${ }^{16}$ Confocal Raman spectroscopy imaging (cRSI) has further been demonstrated as a label-free methodology for biomolecular imaging in zebrafish embryos on a whole-body level. ${ }^{17}$

Despite these advances, the limits in performance of a conventional laboratory source in zebrafish embryo imaging with respect to spatial resolution and sensitivity, have, to the best of our knowledge, so far not been determined. Therefore, we have evaluated the table-top instrument SkyScan1275 (Bruker, Kontich, Belgium) to perform three-dimensional X-ray microscopy of zebrafish embryos. Similar to tomography studies of paraffinembedded brain, ${ }^{18}$ the absorption mode should yield enough contrast to identify many anatomical microstructures. We investigate to what extent anatomical features of zebrafish embryos can be visualized with a conventional laboratory source by employing improved sample preparation and compare our result to publicly available data $^{13}$ taken with $\mathrm{SR} \mu \mathrm{CT}$.

\section{METHODOLOGY}

\subsection{Sample preparation}

For the experiment, the zebrafish embryos were euthanized 72 hours post fertilization (hpf) with tricaine methanesulfonate containing $0.612 \mathrm{mM}$ trisaminomethane. Subsequently, they were fixed at room temperature in $4 \%$ paraformaldehyde and stored at a temperature of $4{ }^{\circ} \mathrm{C}$. Fixed specimens were dehydrated using ethanol at increasing concentrations $(25 \%, 50 \%, 70 \%,>99.5 \%, 15 \mathrm{~min}$ each). For X-ray microtomography, the samples were transferred with a micro-pipette to sample holders consisting of polyimide tubes (Polyimide-Tube AWG 20 NATUR, electrical grade, Rotima AG, Stäfa, Switzerland) with an inner diameter of $0.813 \mathrm{~mm}$ and a wall thickness of $51 \mu \mathrm{m}$. The tubes were further sealed at both ends with BRAND ${ }^{\mathrm{TM}}$ haematocrit sealing compound (Sigma-Aldrich, Wertheim, Germany) and two layers of nail polish. For the paraffin embedding, the embryos fixed in ethanol were washed twice in xylene (>98\%, Carl Roth, Switzerland) and further transferred into standard histological paraffin blocks (Leica paraplast bulk, USA) liquefied at a temperature of $68{ }^{\circ} \mathrm{C}$. After cooling, cylinders were extracted from the paraffin block using a metal punch with an inner diameter of 2.8 or $3.6 \mathrm{~mm}$.

\subsection{Laboratory-based absorption contrast-based micro computed tomography}

The samples were imaged in the commercially available table-top SkyScan $1275 \mu \mathrm{CT}$ scanner (Bruker, Kontich, Belgium), equipped with a a $100 \mathrm{kV}$ microfocus X-ray source with a tungsten target (type: L11871-20) and a $3 \mathrm{Mp}(1944 \times 1536$ pixels $)$ CMOS flat panel detector. The tomography experiments were performed with an effective pixel size of $4.2 \mu \mathrm{m}$, a tube voltage of $15 \mathrm{kVp}$, and a beam current of $156 \mu \mathrm{A}$, corresponding to a power of $2.34 \mathrm{~W}$ at which a focal spot size below $5 \mu \mathrm{m}$ can be provided. A total of 720 projections were taken around $360^{\circ}$, with an exposure time of $2.2 \mathrm{~s}$ and 8 frames averaged, leading to total scan times of $3.5 \mathrm{~h}$. Data processing and reconstruction was performed in an automated fashion using the dedicated NRecon Software (version 1.7.4). 


\subsection{Energy-dispersive X-ray analysis}

The polyimide tubes (Polyimide-Tube AWG 20 NATUR, electrical grade and Polyimide-Tube AWG 23 BLACK, medical grade) were further investigated by energy-dispersive X-ray (EDX) analysis. Sections of the samples were cut in half, glued onto carbon tape and then a sputtered in a Q300T D sputter coater (Quorum, Laughton, UK) for $100 \mathrm{~s}$ at $80 \mathrm{~mA}$, resulting in a few nanometer thick gold layer. The EDX spectra were taken with a EM-30AXN scanning electron microscope (Coxem, Daejeon, Korea) in high-vacuum mode with an acceleration voltage of $20 \mathrm{kV}$ and a working distance of 10.4 to $10.9 \mathrm{~mm}$.

\section{RESULTS}

\subsection{Sample container characterization}

For X-ray $\mu \mathrm{CT}$ of ethanol embedded zebrafish embryos, polyimide tubes were chosen as sample container in order to fulfil the criteria of being small in diameter, having a cylindrical shape and being highly radiotranslucent. However, the tubes with an inner diameter (ID) of $0.813 \mathrm{~mm}$, which matches the diameter of the zebrafish embryos, exhibited a highly X-ray absorbing layer on the tubes' inner surface. To compare the absorption properties of the two tube candidates (Tube 1: ID $0.813 \mathrm{~mm}$, electrical grade; Tube 2: ID $0.570 \mathrm{~mm}$, medical grade), they were simultaneously imaged in the Skyscan $1275 \mu \mathrm{CT}$ system. Figure 1 shows a comparison of the two tubes. On the top left a vertical slice of Tube 1 and Tube 2 glued on top of each other is shown. In the horizontal cross sections, taken at the positions indicated in blue (Tube 1) and red (Tube 2) it is clearly visible that while Tube 1 exhibits a bright, X-ray absorbing layer in its internal wall, the absorption of Tube 2 is homogeneous. This difference in X-ray absorbance was further visualized by radial absorption profiles of the tubes, shown in the bottom part of Figure 1. The data shows that the highly absorbing layer in Tube 1 amounts to approximately twice the absorption of pure polyimide.

To determine the reason for the high-absorbing coating, the surface structure and material composition of the inner wall of the tubes was investigated by a combination of scanning electron microscopy with energy dispersive X-ray spectroscopy. Microscopic inspection with a SEM already revealed differences in appearance between the tubes' inner surfaces: while Tube 1 with the highly absorbing layer showed inhomogenities on the micrometer scale and some cracks ( $c f$. Fig. 2, Top left), Tube 2 had a homogeneous surface with less features ( $c f$. Fig. 2, Top right). By subsequent EDX analysis, which is shown in the bottom part of Figure 2, the following peaks were observed for Tube 1: oxygen (K $\alpha: 525 \mathrm{eV})$, aluminium $(\mathrm{K} \alpha: 1.486 \mathrm{keV})$, silicon $(\mathrm{K} \alpha: 1.739 \mathrm{keV})$, copper $(\mathrm{K} \alpha$ : $8.040 \mathrm{keV}, \mathrm{K} \beta: 8.905 \mathrm{eV}, \mathrm{L} \alpha: 930 \mathrm{eV})$ and for Tube 2: carbon $(K \alpha: 277 \mathrm{eV})$, oxygen $(K \alpha: 525 \mathrm{eV})$, aluminium $(K \alpha: 1.486 \mathrm{keV})$ and silicon $(\mathrm{K} \alpha: 1.739 \mathrm{keV})$. Interpreting those peaks, the EDX peak profile of Tube 2 matches well for what is expected for kapton polyimide films with a chemical formula of $\mathrm{C}_{22} \mathrm{H}_{10} \mathrm{~N}_{2} \mathrm{O}_{5}$. The nitrogen peak $(\mathrm{K} \alpha: 392 \mathrm{eV})$ is not observed due to the limited energy resolution, as this peak lies in between the $\mathrm{C}$ and $\mathrm{O}$ $\mathrm{K} \alpha$-line. From the peak profile of Tube 1, the contamination layer can be identified as copper in an oxidized state. 

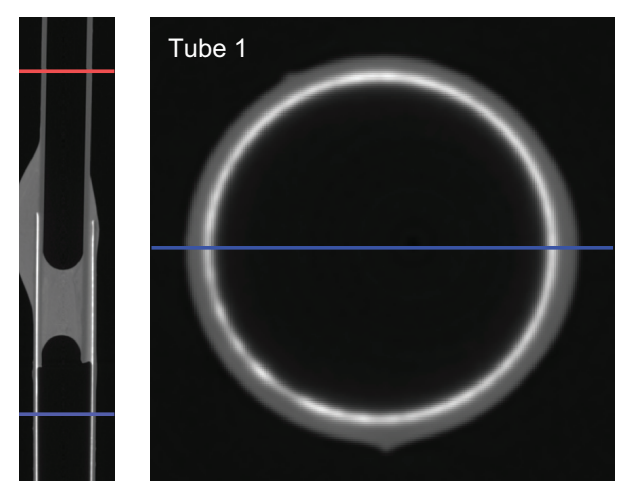

$1 \mathrm{~mm}$

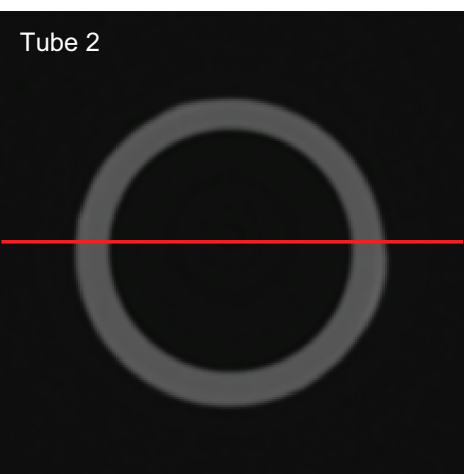

$0.2 \mathrm{~mm}$

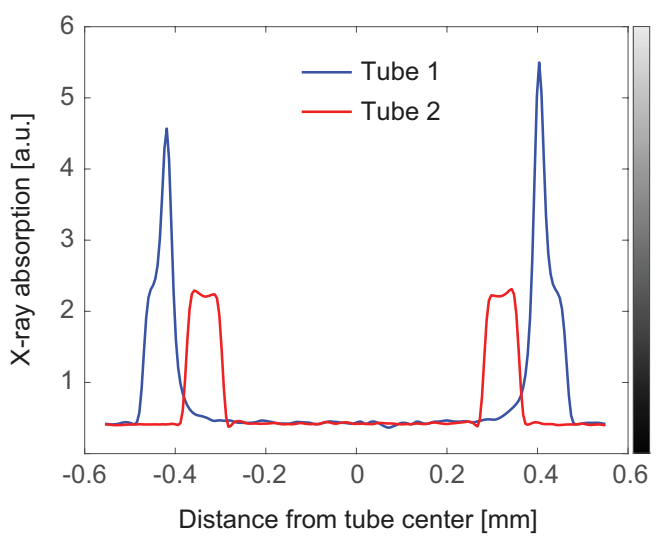

Figure 1. X-ray microtomography measurements of polyimide tubes. Top: Vertical slice of two tubes glued together: ID $0.570 \mathrm{~mm}$, electrical grade (Tube 1) and ID $0.813 \mathrm{~mm}$, medical grade (Tube 2). Horizontal cross sections taken at the positions indicated in blue (Tube 1) and red (Tube 2). Bottom: X-ray absorption profile along the lines marked in the horizontal cross sections.

\subsection{Revealing zebrafish embryo anatomy with a conventional $\mu \mathrm{CT}$ system}

For the experimental data shown in this work, zebrafish embryos were imaged at a time point 72 hours post fertilization, a development stage which is referred to as the protruding mouth stage. In order to better understand the identified anatomical structures within this developmental time-window, this section is introduced by a brief insertion on zebrafish embryogenesis.

Zebrafish undergo a very rapid embryogenesis that can be subdivided into seven broad periods (zygote, cleavage, gastrula, segmentation, pharyngula and hatching period) which occur during the first three days after fertilization. ${ }^{19}$ Already in the pharyngula period (24-48 hpf) the zebrafish embryo is a bilaterally organized creature which possesses the classic vertebrate bauplan. It has a well-developed notochord just ventral to the neural tube, a completed set of somites (in total 30-34 pairs), and the brain is sculptured into five lobes. The pharyngeal arches develop rapidly from a primordial region and the circulatory system forms. In the subsequent hatching period (48-72 hpf) a rapid development of the pectoral fins, the jaws and the gills is observed. Further, at the end of this period, prominent changes occur in the pharyngeal region: the mouth opens and it is repositioned anteriorward due to jaw morphogenesis. Finally, in the protruding mouth stage around $72 \mathrm{hpf}$ - as the name implies - the mouth wide open and protrudes anteriorly just beyond the eye. Further, the blade of the pectoral fin continues to expand and the gill slits become visible. With this background knowledge on zebrafish embryogenesis, we will present and discuss next, what features were identified within our $\mu \mathrm{CT}$ measurement data. 

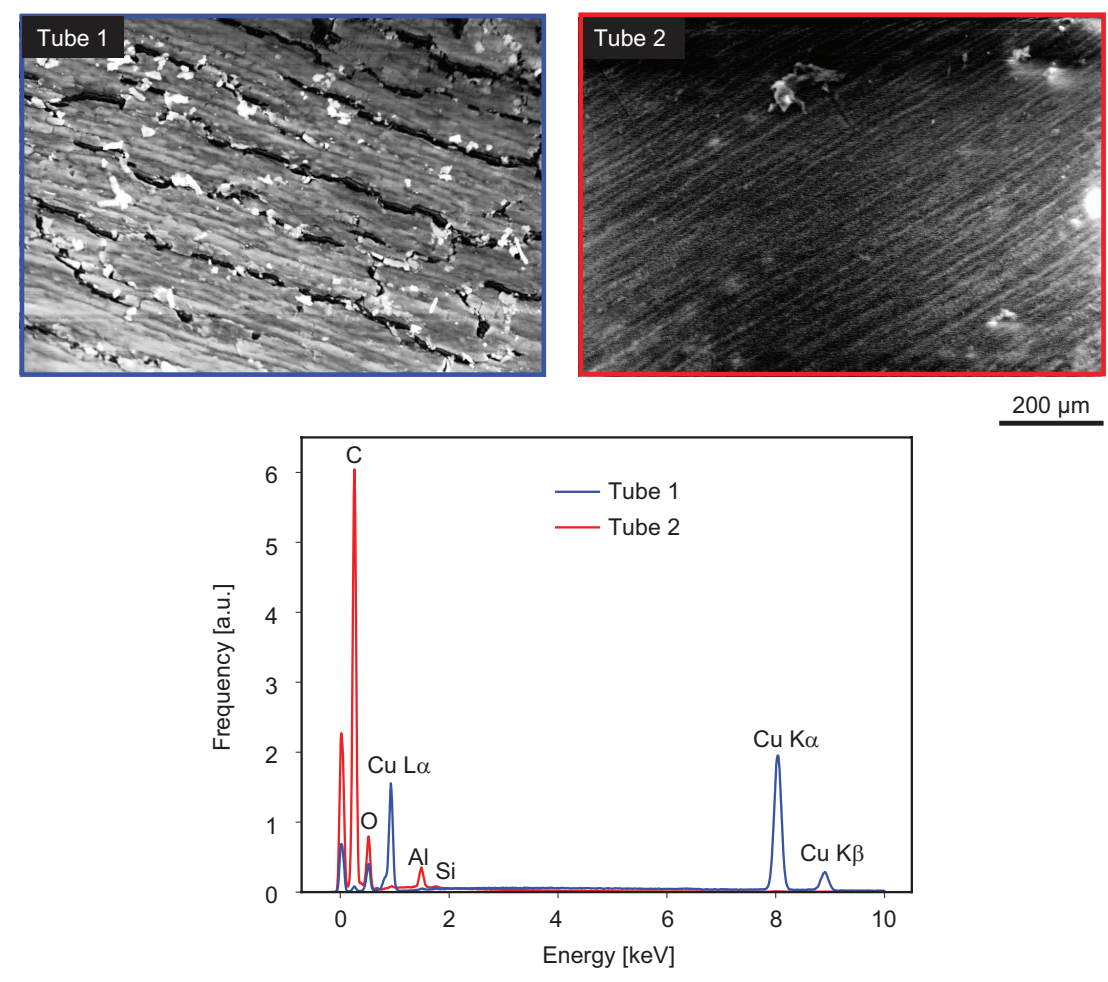

$200 \mu \mathrm{m}$

Figure 2. Investigation of the polyimide tubes with a scanning electron microscope. Top: SEM images of the inner surfaces of two tubes: ID $0.570 \mathrm{~mm}$, electrical grade (Tube 1); ID $0.813 \mathrm{~mm}$, medical grade (Tube 2). Bottom: Corresponding EDX spectra.

Virtual slices through the $\mu \mathrm{CT}$ volume can reveal a wealth of anatomical features of $72 \mathrm{hpf}$ zebrafish embryos. The measurements here show ethanol- and paraffin-embedded specimens in absorption contrast mode with an effective voxel size of $4.2 \mu \mathrm{m}$, corresponding to an estimated spatial resolution of $13 \mu \mathrm{m}$, determined by the method introduced by Mizutani et al. ${ }^{20}$ In Figure 3 axial cross sections are shown, taken at the positions indicated in the volume rendering. For embedding in ethanol the polyimide tube is artificially colored in light brown. The labels that are newly introduced in the text, are referred to with turquoise colored labels in rounded brackets. Within the head region (Fig. $3 \mathrm{~A} / \mathrm{A}^{\prime}$ ) the eye (2) with the lens (1), the diencephalic part (5) of the brain, the open mouth, leading to the pharynx (4) as well as the mandibular bone (3) are visible. In the pectoral region (Fig. 3 B/B') the embryonic heart with the ventricle (6) and the pectoral sac (7) can be identified. The metencephalic part (8) of the brain is visible and the notochord (9) emerges in that body section. Within the region of the regressing yolk sac (11) (Fig. $3 \mathrm{C} / \mathrm{C}^{\prime}$ ), the bilaterally organized pectoral fins (10) emerge. Throughout the tail (Fig. 3 D/D') the notochord (9), which is flanked by the segmental muscles, the so-called myotomes (13), is very prominent and the outlines of the gut (12) can be seen in the ventral part of the embryo, anterior to the cloaca. 


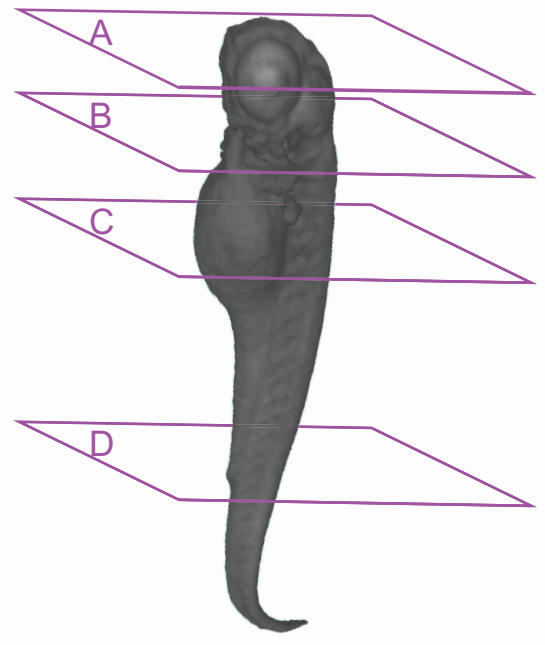

ethanol

A

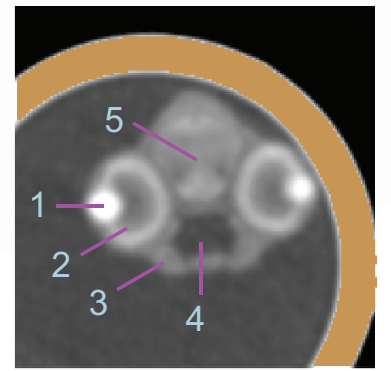

B

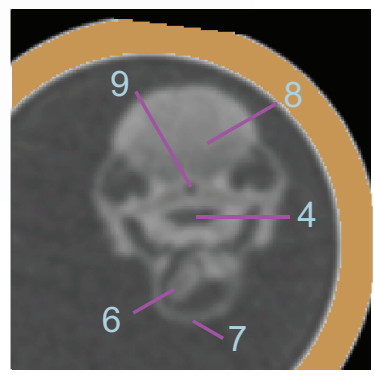

\section{paraffin}

$A^{\prime}$

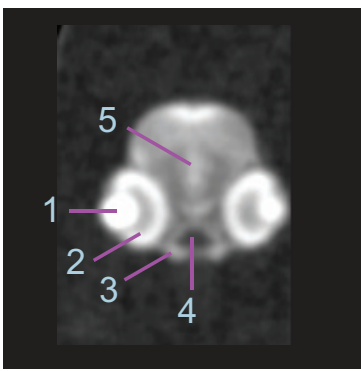

1 Lens

2 Eye

3 Mandibular bone

4 Pharynx

5 Diencephalon

6 Ventricle

7 Pericardial sac

8 Metencephalon

9 Notochord

10 Pectoral fin

11 Yolk sac

12 Gut

13 Myotomes

(Segmental muscles)
C

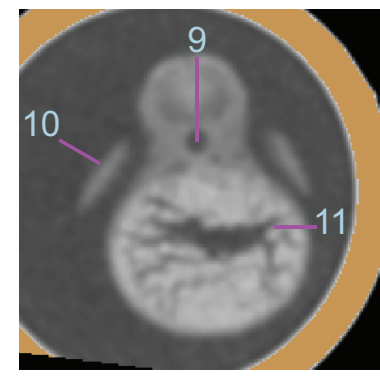

D

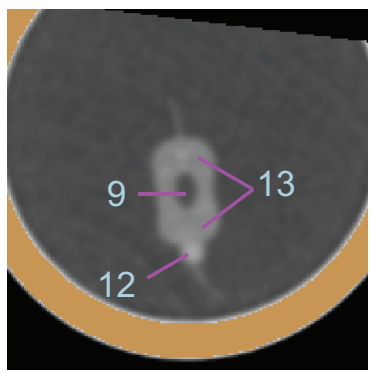

C'
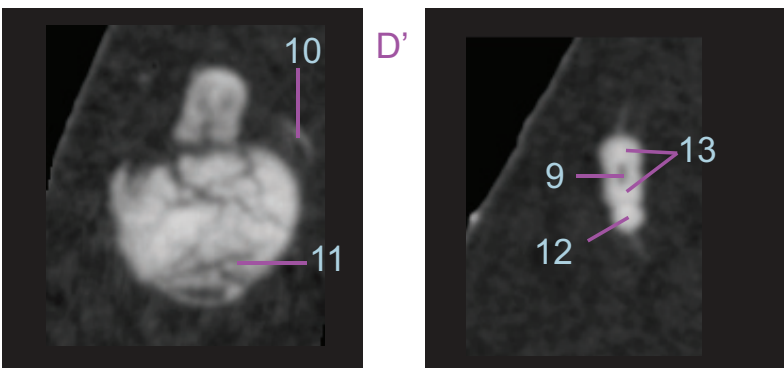

Figure 3. Axial cross sections uncover a dozen anatomical features in $72 \mathrm{hpf}$ larval zebrafish for embedding in ethanol (A-D) and in paraffin (A'-D'). The location of the cross sections in relation to the organism is shown in a 3D volume rendering. The polyimide tube is colored in light brown. Labels for the identified anatomical features are provided in the legend and in the text. 
In sagittal cross sections, shown in Figure 4, several parts of the brain, namely the telencephalon (14), diencephalon (5), metencephalon (8) (Fig. 4 A/A') and the cerebellum (16) (Fig. 4 B) can be localized. In Fig 4 A the swim bladder (15) is visible dorsal to the yolk sac. Within the head, the otoliths (17), which consist of crystalline calcium carbonate, are revealed (Fig. 4 B).
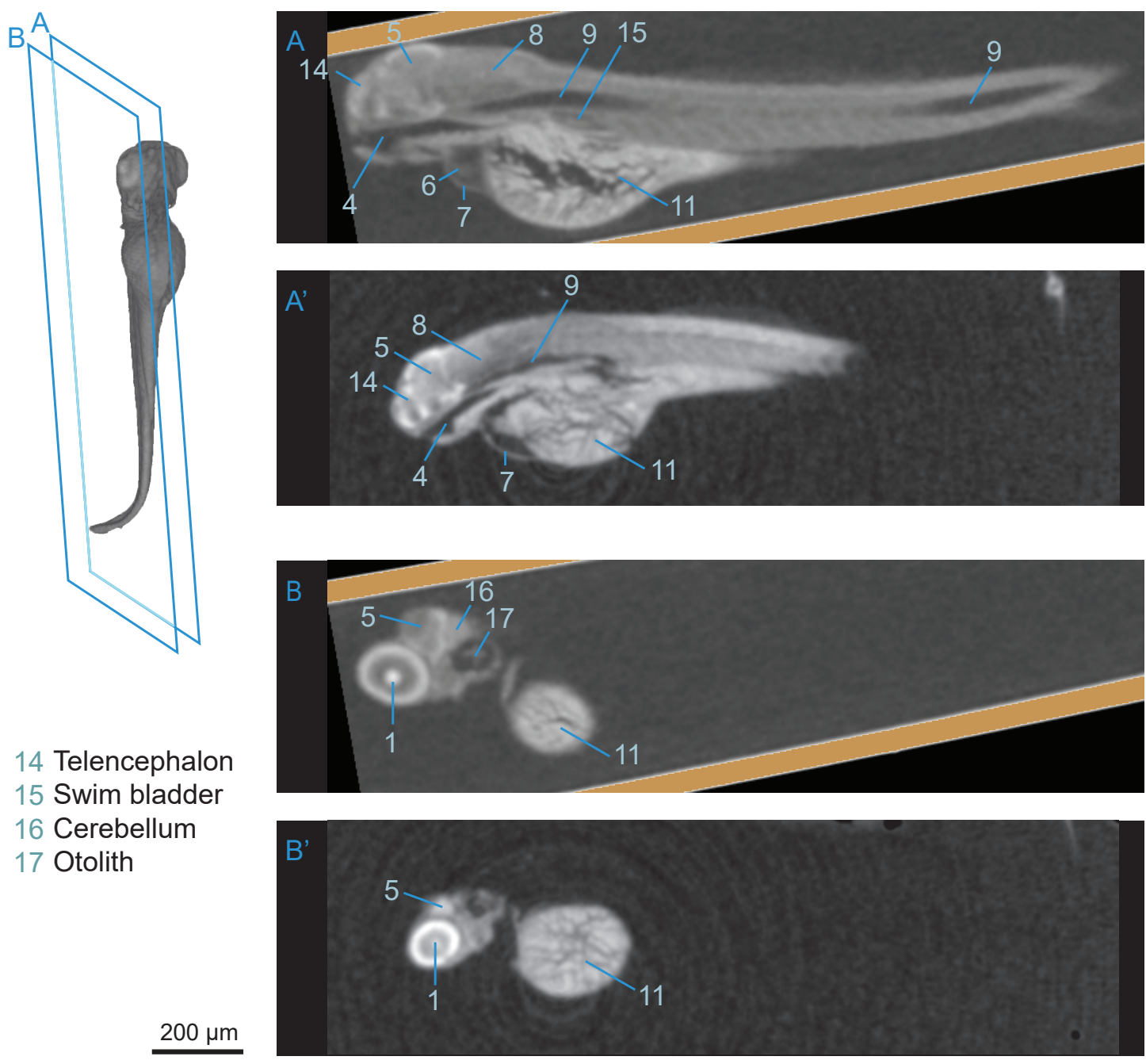

Figure 4. Sagittal cross sections highlight further anatomical features of $72 \mathrm{hpf}$ larval zebrafish for embedding in ethanol $(\mathrm{A}, \mathrm{B})$ and in paraffin $\left(\mathrm{A}^{\prime}, \mathrm{B}^{\prime}\right)$. The location of the cross sections in relation to the organism is shown in a 3D volume rendering. The polyimide tube is colored in light brown. The additional labels are designated in the legend and in the text. 
In coronal cross sections, shown in Figure 5, further structures belonging to the neural system are visible. However, they are relatively hard to delimit and some of them could not be unambiguously assigned. The hindbrain ventricle (18), the olfactory bulb (19) and the hypothalamus (20) were located and within the framed regions also other structures emerge, which could belong to the epiphysis, the pallium or the eminentia thalamy. Again, the pectoral fins (10) are very prominent (Fig. 5 A, B') and the notochord (9) spans the entire posterior part of the zebrafish embryo.

\subsection{Feature extraction with respect to synchrotron radiation-based tomography}

By providing a direct comparison between our laboratory-based $\mu \mathrm{CT}$ measurements with the Skyscan 1275 system and a publicly available $\mathrm{SR} \mu \mathrm{CT}$ dataset, ${ }^{13}$ it can be shown which features can be readily identified with a conventional X-ray source and which additional anatomical microstructures can be revealed by employing synchrotron radiation with improved beam properties. SR $\mu \mathrm{CT}$ imaging was performed on ethanol-embedded $72 \mathrm{hpf}$ zebrafish embryos at the TOMCAT X02DA beamline of the Swiss Light Source with a monochromatized beam with a photon energy of $10.25 \mathrm{keV}$ and an effective voxel size of $325 \mathrm{~nm}$, corresponding to a spatial resolution around $2 \mu \mathrm{m}$. To exploit phase contrast, single distance phase retrieval was performed on the SR $\mu \mathrm{CT}$ projections. $^{21}$ In the subsequent figures (Figs. 6 to 8) the previously shown axial, sagittal and coronal slices of the laboratory-based measurement of the ethanol-embedded sample are directly compared to corresponding slices of the SR $\mu \mathrm{CT}$ dataset. In the virtual cuts of these more detailed data, only the anatomical features which are additionally revealed in comparison to the laboratory-based measurement are labelled.
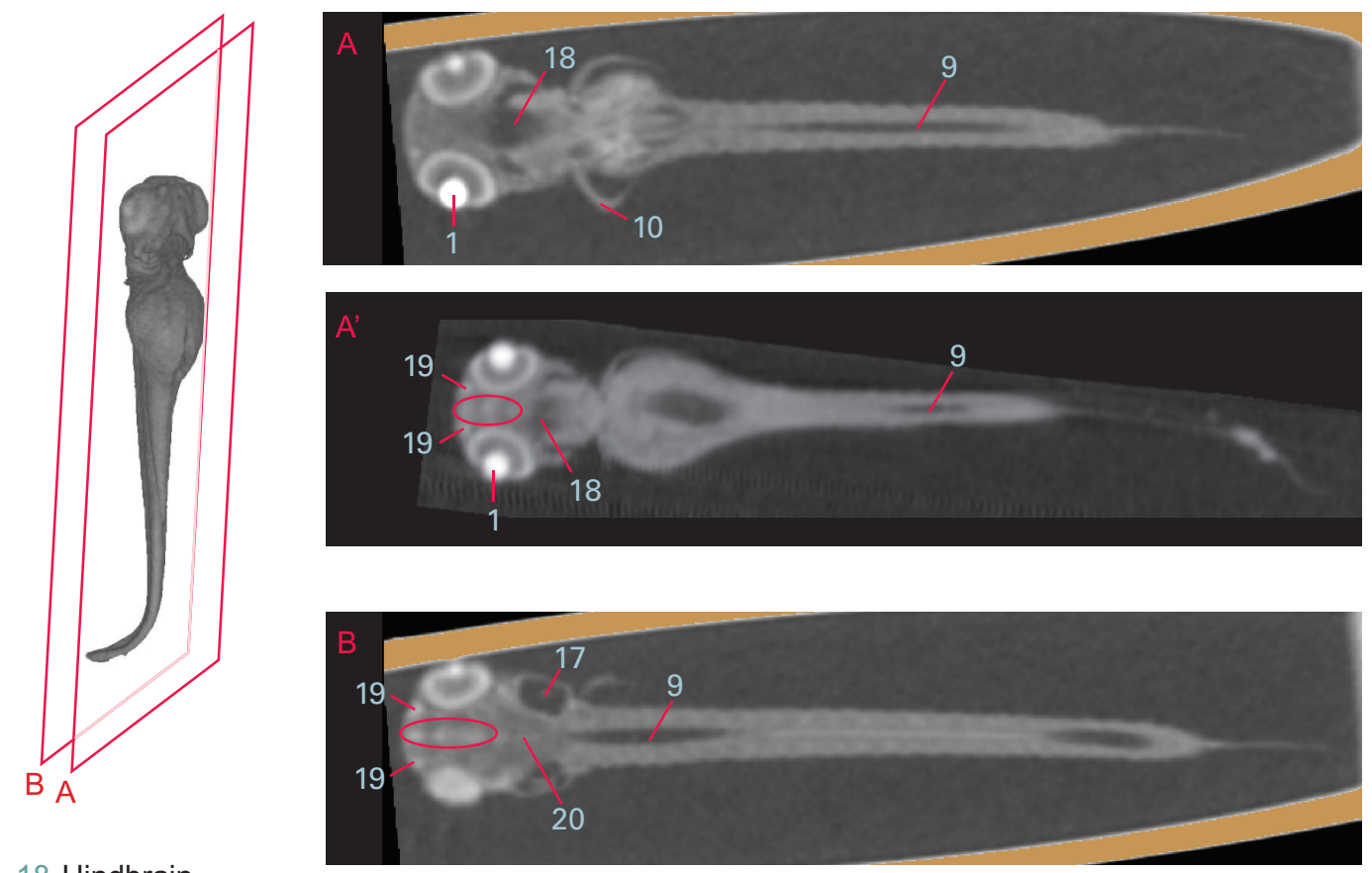
18 Hindbrain ventricle
19 Olfactory bulb
20 Hypothalamus

$200 \mu \mathrm{m}$

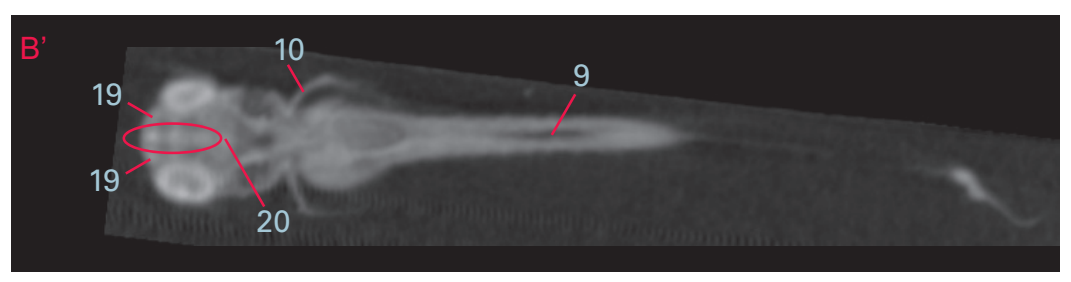

Figure 5. Coronal cross sections highlight further anatomical features of $72 \mathrm{hpf}$ larval zebrafish for embedding in ethanol $(\mathrm{A}, \mathrm{B})$ and in paraffin $\left(\mathrm{A}^{\prime}, \mathrm{B}^{\prime}\right)$. The location of the cross sections in relation to the organism is shown in a $3 \mathrm{D}$ volume rendering. The polyimide tube is colored in light brown. The additional labels are designated in the legend and in the text. 
For the axial view, shown in Figure 6, the individual cell layers of the eye, namely the outer plexiform layer (21), the inner plexiform layer (24) as well as the ganglion cell layer (23), and also the iris (22) can be identified with cellular resolution (Fig. 6 A'). Further, the trabecula (25) are visible above the zebrafish mouth. In Figure 6 B' the midbrain tegmentum (26), the macula (27), the hyosymplectic (28) as well as the parachordal basal cartilage (29) are revealed, and the high-density otoliths (17) are very prominent. In Figure 3 C' the liver (31) and the gut (13) can be distinguished dorsal of the yolk sac (11), as well as the spinal chord (32) which lies dorsal of the notochord (9). Further, the pronephric duct (31) of the embryonic nephron is visible in this sectional plane. Within the tail region (Fig. 6 D') the individual muscle segments (13) can be clearly distinguished and the spinal chord (32) remains visible.

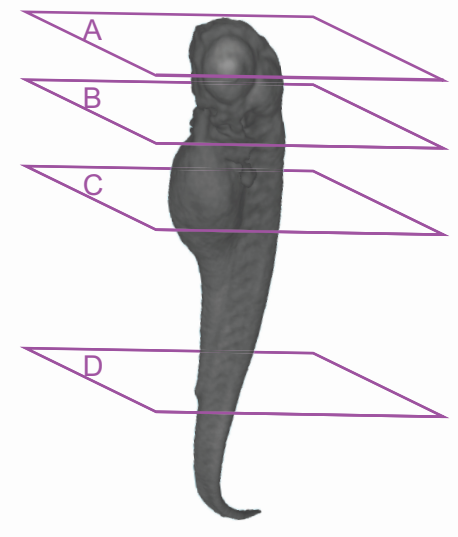

Skyscan 1275

A

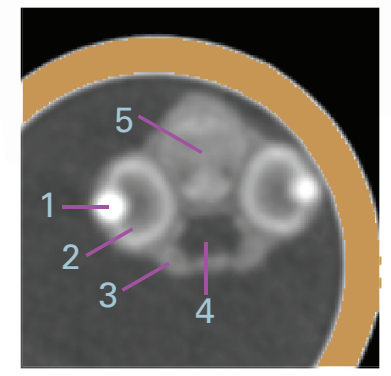

TOMCAT SLS

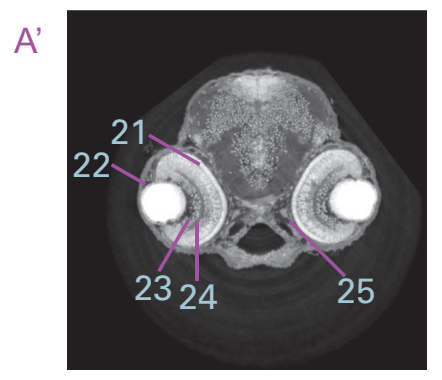

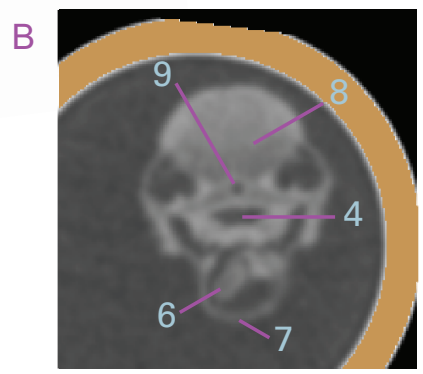

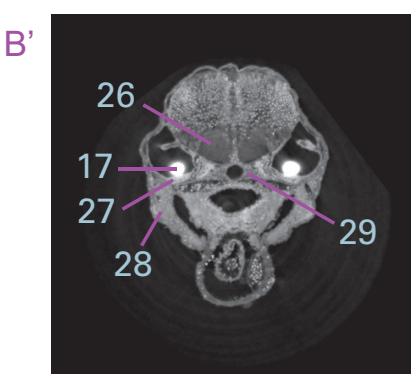

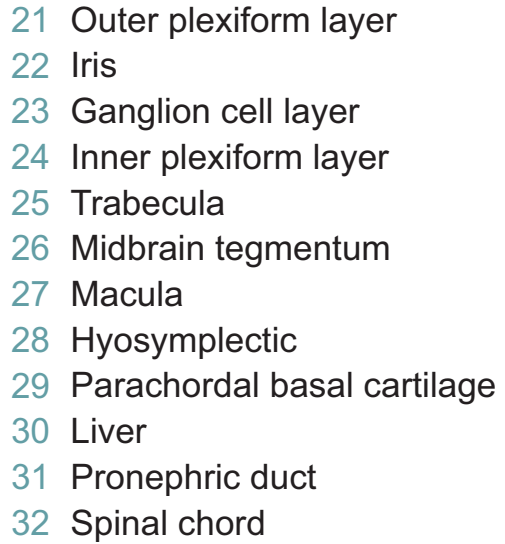

32 Spinal chord
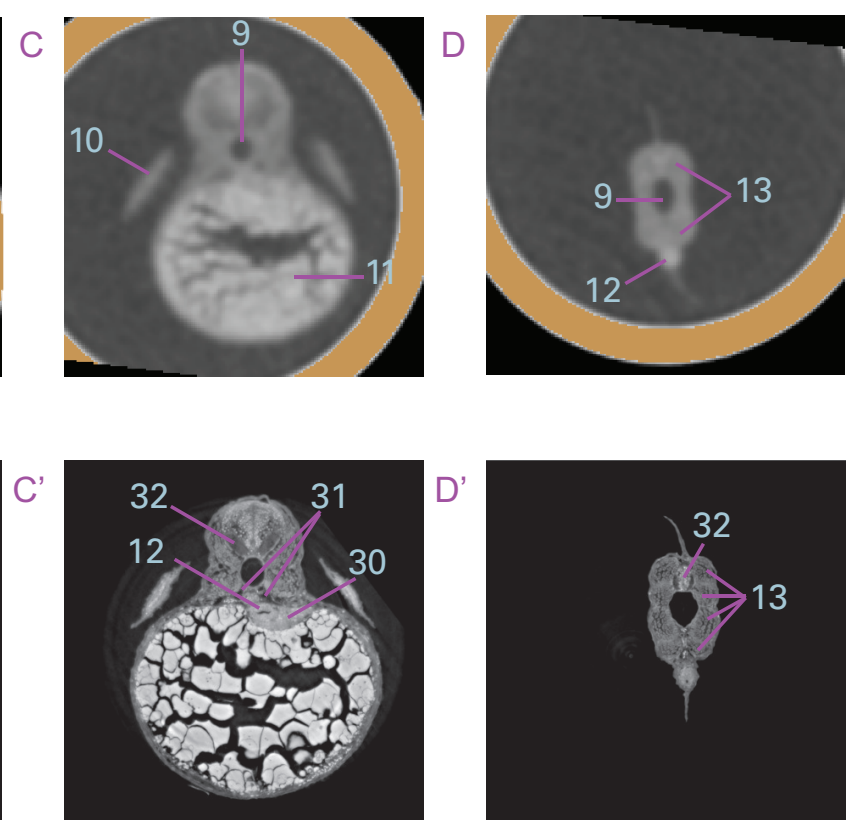

Figure 6. Comparison of laboratory-based X-ray $\mu \mathrm{CT}$ with measurements taken at the Swiss Light Source. ${ }^{13}$ Axial cross sections are shown for embedding in ethanol imaged with the Skyscan 1275 system (A-D) and at the TOMCAT beamline of the Swiss light source (A'-D'). For the SR $\mu$ CT slices (A'-D') only the extra features are labelled. The additional labels are designated in the legend. 
In the sagittal view (Fig. 7) some extra features are revealed: within the brain the medulla oblongata (33) and the epiphysis (35) are visible and the hypophysis (20) can be clearly distinguished. Within the mouth region the pharyngeal arches (34) are prominent and the trabecula (25) can be identified (Fig. 7 A').

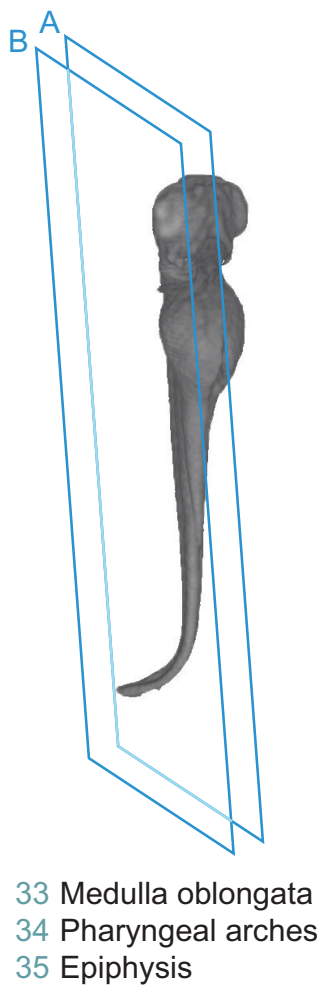

$200 \mu \mathrm{m}$
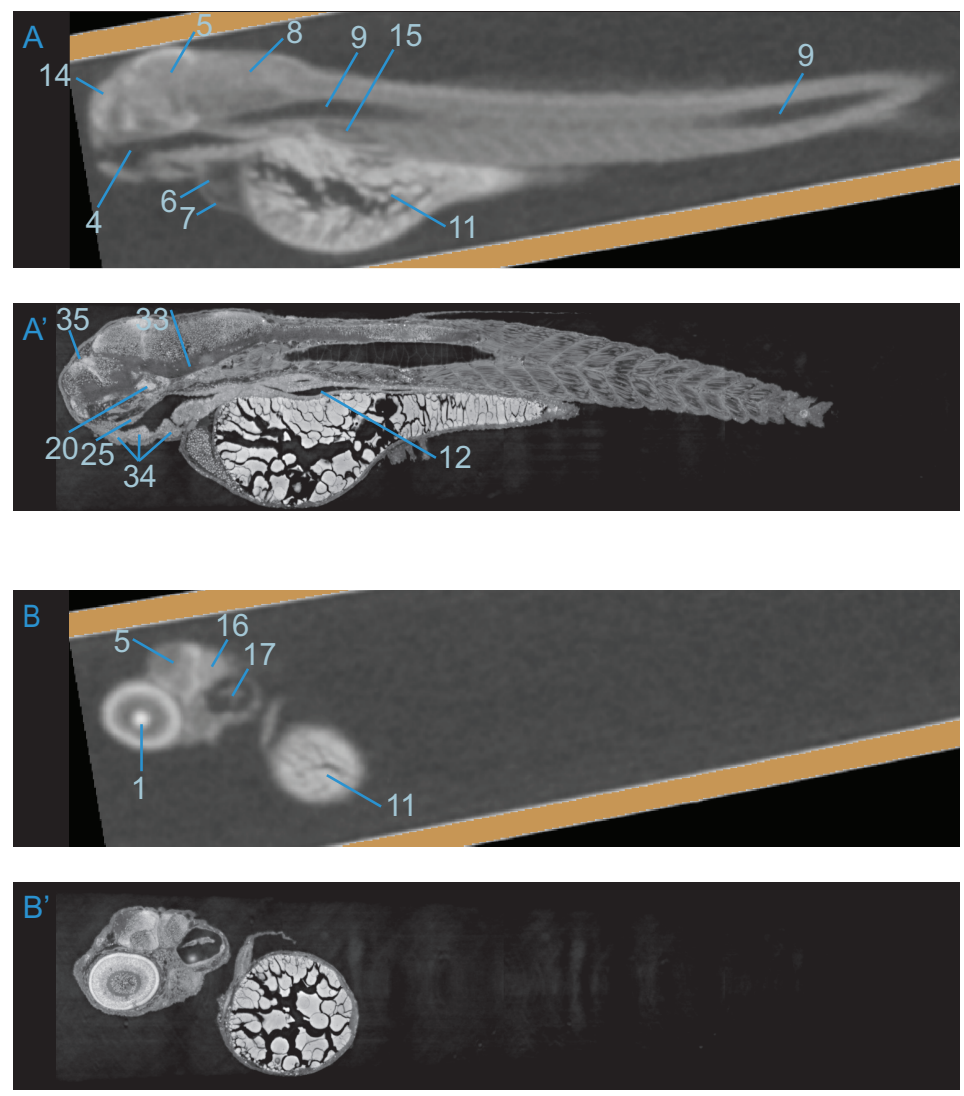

Figure 7. Comparison of laboratory- based X-ray $\mu \mathrm{CT}$ with measurements taken at the Swiss Light Source. ${ }^{13}$ Sagittal cross sections are shown for embedding in ethanol imaged with the Skyscan 1275 system (A, B) and at the TOMCAT beamline of the Swiss light source (A', B'). For the SR $\mu$ CT slices (A', B') only the extra features are labelled. The additional labels are designated in the legend.

Finally, in the coronal view (Fig. 8) the individual layers of the eye, namely the outer (21)- and inner plexiform layer (24) as well as the iris (23) can be identified again (Fig. 8 A') and in addition to the hindbrain ventricle (18) and the olfactory bulb (19) also other brain regions emerge (Fig. 8 A'). 

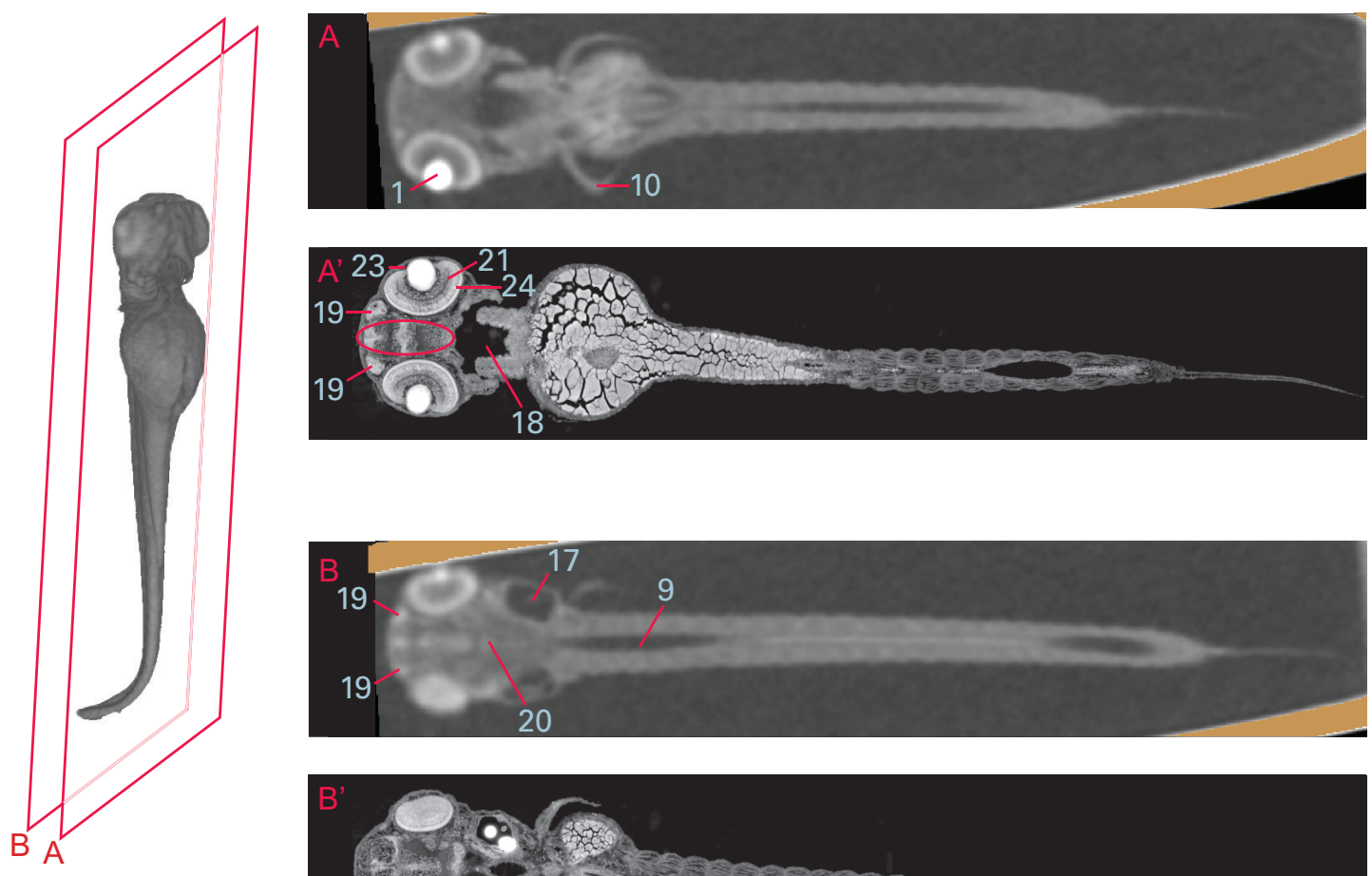

$200 \mu \mathrm{m}$

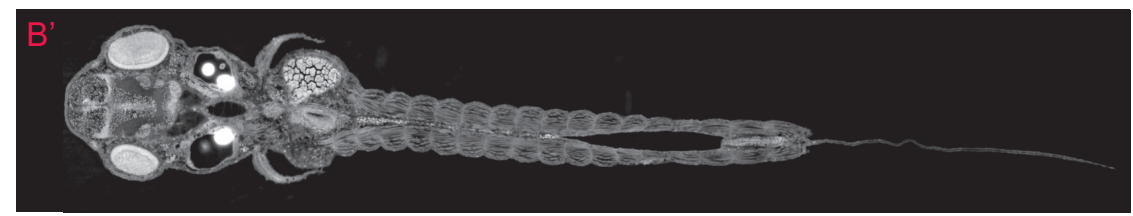

Figure 8. Comparison of laboratory- based X-ray $\mu \mathrm{CT}$ with measurements taken at the Swiss Light Source. ${ }^{13}$ Coronal cross sections are shown for embedding in ethanol imaged with the Skyscan 1275 system (A, B) and at the TOMCAT beamline of the Swiss light source (A', B'). For the SR $\mu \mathrm{CT}$ slices (A', B') only the extra features are labelled.

The strength of $\mu \mathrm{CT}$ in order to phenotype full tissue volumes is further illustrated by video material, showing sets of virtual cross sections through the $72 \mathrm{hpf}$ zebrafish embryos ( $c f$. video 1- 3). These histotomography datasets with isotropic voxel-sizes allow for histology-like phenotyping as well as quantitative analysis. In video 1, showing the laboratory-based measurement of the ethanol-embedded specimen, the sample container, being relatively high-absorbing, is quite prominent. The paraffin-embedded specimen, shown in video 2, however, can be well-distinguished from the embedding material. By employing $\mathrm{SR} \mu \mathrm{CT}$, as demonstrated in video 3, anatomical features can be revealed on a full-organism level with cellular resolution.

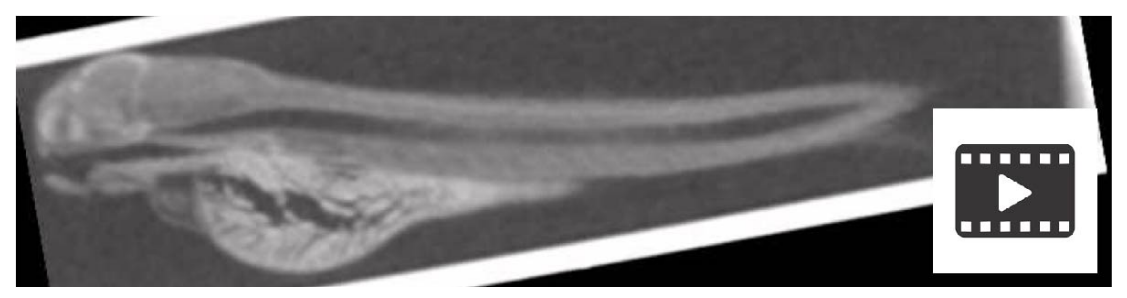

Video 1. Digital larval flipbook of ethanol-embedded $72 \mathrm{hpf}$ zebrafish embryos imaged with the table top $\mu \mathrm{CT}$ scanner. The video shows full cross sections of the larval zebrafish in sagittal orientation with $4.2 \mu \mathrm{m}$-wide isotropic voxels.

http://dx.doi.org/10.1117/12.2593119.1 


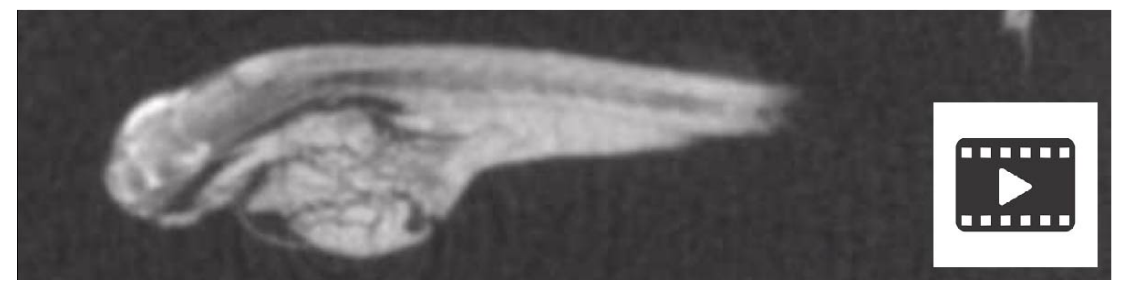

Video 2. Digital larval flipbook of paraffin-embedded $72 \mathrm{hpf}$ zebrafish embryos imaged with the table top $\mu \mathrm{CT}$ scanner. The video shows full cross sections of the larval zebrafish in sagittal orientation with $4.2 \mu \mathrm{m}$-wide isotropic voxels. http://dx.doi.org/10.1117/12.2593119.2

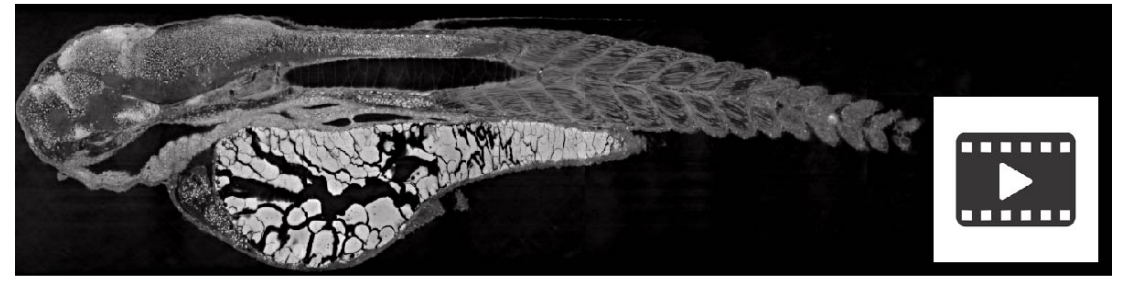

Video 3. Digital larval flipbook of ethanol-embedded 72 hpf zebrafish embryos imaged at the Swiss Light Source. The video shows a selection of 100 full cross- sections throughout the larval zebrafish in sagittal orientation with $0.325 \mu \mathrm{m}$-wide voxels. http://dx.doi.org/10.1117/12.2593119.3

\section{DISCUSSION}

$\mathrm{X}$-ray $\mu \mathrm{CT}$ is a well-established and powerful imaging technique due to the penetration depth of hard X-rays that enables whole-organism imaging with micrometer resolution without relying on staining or physical slicing. This allows for dynamic reslicing of the whole organism and three-dimensional renderings of entire organs or cell patterns. The histotomographic approach can be used to develop detailed morphological lifespan atlases or to segment full organs and organ changes during development or disease progression. This approach has been readily demonstrated, e.g. by providing a volume rendering of the gastrointestinal tract of 33 dpf juvenile zebrafish and for quantifications of brain cell nuclei. ${ }^{12}$ In this tomography-based study we have investigated how the performance of conventional electron-impact X-ray sources compares to synchrotron radiation sources for imaging zebrafish embryos. Despite being severely limited in beam properties with regard to brightness, tunabilty, as well as temporal coherence, we have observed that imaging with conventional electron-impact X-ray sources can already provide three-dimensional data for the identification of a wealth of anatomical features.

Being limited in beam properties makes optimization of sample embedding techniques even more important. As expected, embedding in ethanol and paraffin both comprise a set of challenges. For ethanol embedding, the choice of a suitable container is crucial to immobilize the specimen while interfering as little as possible with the overall X-ray absorption. Paraffin embedding overcomes the need for a container, however, heat damage of the tissue becomes an issue and appropriate shaping of the specimen can be challenging. Each embedding technique comes along with its specific soft-tissue contrast. In this work, we have observed a comparable performance of both embeddings for highlighting the zebrafish anatomy ( $c f$. Figure 3-5). Image registration between the embeddings could provide a quantitative comparison between ethanol- and paraffin-embedded zebrafish embryos with respect to image contrast in specific body parts, and could further reveal hidden features by combining the information of both embeddings as previously demonstration for the combination of histology and $\mu \mathrm{CT}$ data. ${ }^{22}$ Preliminary results have readily shown that there is a correspondence between the manually selected virtual cross sections, but image registration between embeddings and also between laboratory-based and synchrotron radiation-based $\mu \mathrm{CT}$ is still part of ongoing investigations and will be presented in an upcoming SPIE conference.

A constraint of this study was that the Skyscan 1275 system provides only absorption contrast. This is typically limited in soft tissues because sensitivity depends linearly on tissue density and by a power law on the atomic number. Nevertheless, using an acceleration voltage of $15 \mathrm{kVp}$ offered sufficient contrast to identify 
several internal organs of the zebrafish larvae, although often it was impossible to precisely delimit the structures from their surroundings. Imaging at even lower X-ray energies would provide improved soft-tissue contrast, however, we were limited here to the operation window of the Skyscan 1275 system. The estimated spatial resolution of $13 \mu \mathrm{m}$ was insufficient to visualize individual biological cells within the zebrafish embryo.

In contrast, synchrotron radiation-based imaging offers phenotyping of whole organisms with single cell resolution. Synchrotron radiation provides high brightness, flux and tunability, allow for improved spatial and density resolution. Improved density resolution is mainly due to the higher X-ray flux which gives rise to faster acquisition times and better photon statistics, and the high spatial and temporal coherence of the X-ray beam, which can be used for phase contrast-based imaging. At low X-ray energies, phase contrast-based imaging, which directly relates to the specimen's electron density, is orders of magnitude more sensitive than absorption contrast. This is in agreement with the high soft-tissue contrast observed in the synchtrotron dataset, which allowed for the identification of the full zebrafish-anatomy down to the individual cell. This single-cell resolution is e.g. impressively visualized within e.g. the eye, as we see in Figure 6 A' and Figure 8 A'.

There remains a performance gap between between conventional laboratory X-ray sources and synchrotron radiation sources. This gap could be bridged by advanced X-ray source technologies such as inverse Compton scattering sources, ${ }^{23}$ as in operation at the Munich Compact Light Source. ${ }^{24}$ Such an advanced X-ray source provides synchrotron-like beam properties, offering high flux, tunability and some degree of monochromaticity while fitting into a laboratory and thereby provides accessibility for the local users. ${ }^{25}$

Nevertheless, despite being limited in spatial and density resolution with laboratory-based $\mu \mathrm{CT}$, still several research questions could be addressed with the comparatively simple $\mu \mathrm{CT}$ scanner: e.g. developmental staging is possible by identification of many anatomical features and morphological changes can be monitored. Potentially this approach could also be used to e.g. locate clusters of injected nanoparticles or other medically relevant nanomaterials, as previously shown for the synchrotron-based approach, ${ }^{13}$ based on their strong difference in attenuation contrast with respect to the soft tissues.

\section{CONCLUSION}

$\mathrm{X}$ rays belong to the probes of choice for three-dimensional visualization of paraffin- and ethanol-embedded tissue samples without physical slicing. Whereas synchrotron radiation-based $\mu \mathrm{CT}$ data allowed for the visualization of microanatomical features in great detail, a simple to operate and cost-effective table-top $\mu \mathrm{CT}$ system enabled us to identify about two thirds of these structures at reduced resolution. We suggest that laboratory-based $\mu \mathrm{CT}$ systems can be used for clinical applications such as routine diagnostic evaluations of biopsies. Furthermore, pilot studies using readily available table-top $\mu \mathrm{CT}$ instruments can provide valuable insights when it comes to the planning and support of costly synchrotron-based imaging experiments.

\section{ACKNOWLEDGMENTS}

The project was financially supported by the Swiss National Science Foundation (Project No. 185058). The authors acknowledge the usage of the electron microscope with EDX equipment (EM-30AXN Coxem, Daejeon, Korea) provided free of charge by Gloor Instruments (Kloten, Switzerland). They are especially grateful to Dr. Iwan Jerjen for his active support.

\section{REFERENCES}

[1] Lele, Z. and Krone, P. H., "The zebrafish as a model system in developmental, toxicological and transgenic research," 14(1), 57-72 (1996).

[2] Stanton, M. F., "Diethylnitrosamine-Induced Hepatic Degeneration and Neoplasia in the Aquarium Fish, Brachydanio rerio," JNCI: Journal of the National Cancer Institute 34(1), 117-130 (1965).

[3] Campbell, F., Bos, F. L., Sieber, S., Arias-Alpizar, G., Koch, B. E., Huwyler, J., Kros, A., and Bussmann, J., "Directing Nanoparticle Biodistribution through Evasion and Exploitation of Stab2-Dependent Nanoparticle Uptake," ACS Nano 12(3), 2138-2150 (2018). 
[4] Sieber, S., Grossen, P., Detampel, P., Siegfried, S., Witzigmann, D., and Huwyler, J., "Zebrafish as an early stage screening tool to study the systemic circulation of nanoparticulate drug delivery systems in vivo," Journal of Controlled Release 264, 180-191 (2017).

[5] Witzigmann, D., Uhl, P., Sieber, S., Kaufman, C., Einfalt, T., Schöneweis, K., Grossen, P., Buck, J., Ni, Y., Schenk, S. H., Hussner, J., Meyer zu Schwabedissen, H. E., Québatte, G., Mier, W., Urban, S., and Huwyler, J., "Optimization-by-design of hepatotropic lipid nanoparticles targeting the sodium-taurocholate cotransporting polypeptide," eLife 8 (2019).

[6] Nishimura, Y., Inoue, A., Sasagawa, S., Koiwa, J., Kawaguchi, K., Kawase, R., Maruyama, T., Kim, S., and Tanaka, T., "Using zebrafish in systems toxicology for developmental toxicity testing," $\mathbf{5 6}(1), 18-27$ (2016).

[7] Howe, K., Clark, M. D., Torroja, C. F., Torrance, J., and Berthelot, C., "The zebrafish reference genome sequence and its relationship to the human genome," Nature 496(7446), 498-503 (2013).

[8] Peng, K., Tomatsu, I., Korobko, A. V., and Kros, A., "Cyclodextrin-dextran based in situ hydrogel formation: A carrier for hydrophobic drugs," Soft Matter 6(1), 85-87 (2009).

[9] Karlsson, J., Von Hofsten, J., and Olsson, P. E., "Generating transparent zebrafish: A refined method to improve detection of gene expression during embryonic development," Marine Biotechnology 3(6), 522-527 (2001).

[10] Grunwald, D. J. and Eisen, J. S., "Headwaters of the zebrafish - emergence of a new model vertebrate," 3(9), 717-724 (2002).

[11] Voelker, D., Vess, C., Tillmann, M., Nagel, R., Otto, G. W., Geisler, R., Schirmer, K., and Scholz, S., "Differential gene expression as a toxicant-sensitive endpoint in zebrafish embryos and larvae," Aquatic Toxicology 81(4), 355-364 (2007).

[12] Ding, Y., Vanselow, D. J., Yakovlev, M. A., Katz, S. R., Lin, A. Y., Clark, D. P., Vargas, P., Xin, X., Copper, J. E., Canfield, V. A., Ang, K. C., Wang, Y., Xiao, X., Carlo, F. D., Rossum, D. B., Riviere, P. L., and Cheng, K. C., "Computational 3d histological phenotyping of whole zebrafish by x-ray histotomography," eLife 8 (2019).

[13] Cörek, E., Rodgers, G., Siegrist, S., Einfalt, T., Detampel, P., Schlepütz, C. M., Sieber, S., Fluder, P., Schulz, G., Unterweger, H., Alexiou, C., Müller, B., Puchkov, M., and Huwyler, J., "Shedding Light on Metal-Based Nanoparticles in Zebrafish by Computed Tomography with Micrometer Resolution," Small 16(31), 2000746 (2020).

[14] Ferstl, S., Metscher, B., Müller, M., Allner, S., Dierolf, M., Busse, M., Achterhold, K., Gleich, B., and Pfeiffer, F., "Laboratory-based X-ray NanoCT Explores Morphology of a Zebrafish Embryo," Microscopy and Microanalysis 24(S2), 184-185 (2018).

[15] Vågberg, W., Larsson, D. H., Li, M., Arner, A., and Hertz, H. M., "X-ray phase-contrast tomography for high-spatial-resolution zebrafish muscle imaging," Scientific Reports 5, 16625 (2015).

[16] Bourassa, D., Gleber, S. C., Vogt, S., Yi, H., Will, F., Richter, H., Shin, C. H., and Fahrni, C. J., "3D imaging of transition metals in the zebrafish embryo by X-ray fluorescence microtomography," Metallomics 6(9), 1648-1655 (2014).

[17] Høgset, H., Horgan, C. C., Armstrong, J. P., Bergholt, M. S., Torraca, V., Chen, Q., Keane, T. J., Bugeon, L., J., D. M., Mostowy, S., and Stevens, M. M., "In vivo biomolecular imaging of zebrafish embryos using confocal Raman spectroscopy," Nature Communications 11, 6172 (2020).

[18] Khimchenko, A., Deyhle, H., Schulz, G., Schweighauser, G., Hench, J., Chicherova, N., Bikis, C., Hieber, S. E., and Müller, B., "Extending two-dimensional histology into the third dimension through conventional micro computed tomography," NeuroImage 139, 26-36 (2016).

[19] Kimmel, C. B., Ballard, W. W., Kimmel, S. R., Ullmann, B., and Schilling, T. F., "Stages of Embryonic Development of the Zebrafish," tech. rep. (1995).

[20] Mizutani, R., Saiga, R., Takekoshi, S., Inomoto, C., Naoyo, N., Itokawa, M., Arai, M., Oshima, K., Takeuchi, A., Uesugi, K., Terada, Y., and Suzuki, Y., "A method for estimating spatial resolution of real image in the Fourier domain," Journal of Microscopy 261(1), 57-66 (2016).

[21] Paganin, D., Mayo, S. C., Gureyev, T. E., Miller, P. R., and Wilkins, S. W., "Simultaneous phase and amplitude extraction from a single defocused image of a homogeneous object," Journal of Microscopy 206(1), 33-40 (2002). 
[22] Stalder, A. K., Ilgenstein, B., Chicherova, N., Deyhle, H., Beckmann, F., Müller, B., and Hieber, S. E., "Combined use of micro computed tomography and histology to evaluate the regenerative capacity of bone grafting materials," International Journal of Materials Research 105(7), 679-691 (2014).

[23] Hajima, R., "Status and Perspectives of Compton Sources," in [Physics Procedia], 84, 35-39, Elsevier B.V. (2016).

[24] Eggl, E., Dierolf, M., Achterhold, K., Jud, C., Günther, B., Braig, E., Gleich, B., and Pfeiffer, F., "The Munich Compact Light Source: initial performance measures," Journal of Synchrotron Radiation 23(5), 1137-1142 (2016).

[25] Hornberger, B., Kasahara, J., Gifford, M., Ruth, R., and Loewen, R., "A compact light source providing high-flux, quasi-monochromatic, tunable X-rays in the laboratory," in [Advances in Laboratory-based X-Ray Sources, Optics, and Applications VII], Murokh, A. and Spiga, D., eds., 11110(9), 2, SPIE (2019). 